\title{
Transport Phenomena in High-speed Wall-bounded Flows Subject to Concave Surface Curvature
}

\author{
Ernie Rivera \\ NAVAIR Air Station \\ Patuxent River, MD
}

\author{
Guillermo Araya \\ University of Puerto Rico at Mayagüez \\ Mayagüez, PR \\ araya@mailaps.org
}

\begin{abstract}
Turbulent boundary layers that evolve along the flow direction are ubiquitous. Moreover, accounting for the effects of wall-curvature driven pressure gradient and flow compressibility adds significant complexity to the problem. Consequently, hypersonic spatiallydeveloping turbulent boundary layers (SDTBL) over curved walls are of crucial importance in aerospace applications, such as unmanned high-speed vehicles, scramjets, and advanced space aircraft. More importantly, hypersonic capabilities would provide faster responsiveness and longer range coverage to U.S. Air Force systems. Thus, the acquired understanding of the physics behind high speed boundary layers over curved wall-bounded flows can lead to the development of more efficient control techniques for the fluid flow (e.g., wave drag reduction) and aerodynamic heating on hypersonic vehicle design. In this investigation, a series of numerical experiments is performed to evaluate the effects of strong concave curvature and supersonic/hypersonic speeds (Mach numbers of 2.86 and 5 , respectively) on the thermal transport phenomena that take place inside the boundary layer. The flow solver to be used is based on a RANS approach. Two different turbulence models are compared: the SST (Shear Stress Transport) model by Menter and the standard k- $\omega$ model by Wilcox. Furthermore, numerical results are validated by means of experimental data from the literature (Donovan et al., J. Fluid Mech., 259, 1-24, 1994) for the moderate concave curvature case and a Mach number of 2.86. The present study allows us to initially obtain a first insight of the flow physics for a forthcoming better design of 3D meshes and computational boxes, as part of a more ambitious project that involves Direct Numerical Simulation (DNS) of curved wall-bounded flows in the supersonic/hypersonic regime. The uniqueness of this RANS analysis in concave curved walls can be summarized as follows: (i) study of the compressibility effects on the time-averaged velocity and temperature, (ii) analysis of the influence of different inflow boundary conditions.
\end{abstract}

\section{KEYWORDS}

RANS, turbulent boundary layer, supersonic, hypersonic, wall curvature

\footnotetext{
Permission to make digital or hard copies of all or part of this work for personal or classroom use is granted without fee provided that copies are not made or distributed for profit or commercial advantage and that copies bear this notice and the full citation on the first page. To copy otherwise, or republish, to post on servers or to redistribute to lists, requires prior specific permission and/or a fee. Copyright $\odot$ JOCSE, a supported publication of the Shodor Education Foundation Inc.

(c) 2021 Journal of Computational Science Education https://doi.org/10.22369/issn.2153-4136/12/1/3
}

\section{INTRODUCTION}

Significant research effort has been devoted to high speed flight in the last decades, since it is directly connected to "rapid responsiveness, increased survivability in contested environments and efficient range coverage" from a military perspective, according to Schmisseur [12]. What is more, a Mach 6-aircraft would be able to reach the US West Coast in approximately 23 minutes from the US East Coast [12]. In 2013, the Boeing X-51A Waverider Scramjet prototype was released at 50,000 feet as part of the the fourth and final test flight in the U.S. Air Force program, reaching a Mach number of 5.1, which is enough to fly from New York to London in roughly 75 minutes.

Furthermore, due to the complex geometries associated with these high-speed aircraft (as seen in Figure 1); such as unmanned hypersonic vehicles (e.g., Boeing X-51 WaveRider and NASA X-43), scramjets, and space planes; surface curvature plays a crucial role in the boundary layer physics and aerothermodynamics. This is attributed to the combined effect of pressure gradients and streamline curvature (Spina et al. [13]), which induces extra strain rates to the main shear $(\partial U / \partial y)$ associated with streamline curvature $(\partial V / \partial x)$, pressure gradients $(\partial p / \partial x$ and $\partial p / \partial y)$, and bulk compression or dilatation $(\nabla \cdot \mathrm{V})$ (Donovan et al. [5]). The principal features of curved wall-bounded flows (so-called Görtler flows) are the presence of a centrifugal force and a pressure gradient (i.e., $\partial p / \partial n$ ) acting on the fluid in the wall-normal direction as well as a streamline pressure gradient (i.e., $\partial p / \partial s)$ in curvilinear coordinates $(s, n)$.

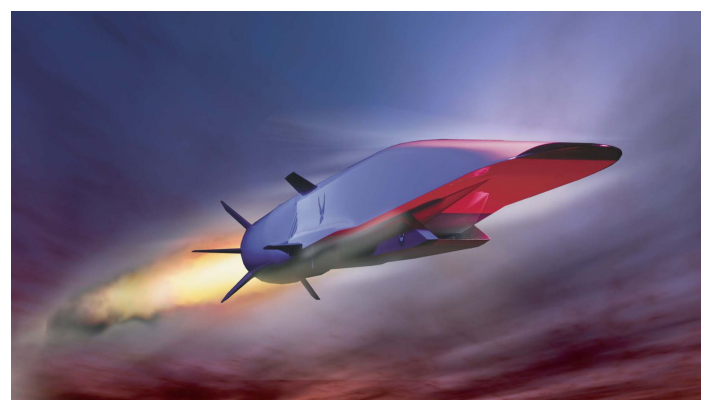

Figure 1: Cartoon of the Boeing X-51A Waverider (source: Wikipedia)

An exhaustive review of pressure gradient and streamline surface curvature effects on the behavior of supersonic turbulent boundary layers can be found in Spina et al. [13]. This revision was focused on experimental studies and two-dimensional geometries, but not on the distortion of compressible turbulent boundary layers by shock 
waves. Furthermore, concave curvature induces a destabilizing effect on the flow by enhancing turbulent mixing, whereas convex curvature is stabilizing. Thomann [17] experimentally studied the isolated effects of wall curvature surfaces (concave and convex) on the heat transfer (Stanton number) in Mach-2.5 turbulent boundary layers by eliminating the streamwise pressure gradients. He found an increase of $20 \%$ on Stanton numbers along the concave wall, while an analogous decrease was observed for the convex wall. Historically, the combined effect of concave surface and Adverse Pressure Gradient (APG) on supersonic/hypersonic turbulent boundary layers has been the motivation of several experimental studies in curved 2D ramps and flared cones, for instance [9] [6] [14] [15] [8]. Smits and his colleagues at Princeton University have performed a series of experiments in two-dimensional concave walls with the objective of gaining insight into the effects of different radii of curvature and turning angles on adverse pressure gradient strength and bulk compression [16] [7] [5]. Furthermore, the typical manifestation of destabilizing effects in concave surfaces in subsonic flow is the generation of Taylor-Görtler-type (T-G) vortices, even in turbulent flows [3] [13]. Jayaram et al. [7] observed significant increases of turbulence levels, structural parameters (such as the stress ratio), and length/time scales of turbulent motions in the larger-curvature case. Donovan et al. [5] found a significant amplification of the Reynolds stresses, and the streamwise length of the average large-scale motions approximately doubled in concave surfaces at a Mach number of 2.86. As concluded in [7] and [5], concave curvature provokes an increase of the wake strength and a dip below the log law in the mean streamwise velocity as well as an emergence of an outer secondary peak on the streamwise component of the Reynolds normal stresses $\left(\overline{u^{\prime 2}}\right)$. These features are attributed to the presence of a streamwise APG on the flow provoked by the concave surface. Similar peculiarities were reported by Araya et al.[2] and Araya and Castillo [1] in incompressible turbulent boundary layers subject to moderate and strong streamwise APG on flat surfaces.

In summary, the performed literature review has revealed the strong influence of wall-curvature driven pressure gradients on the mean flow and heat transfer inside a compressible SDTBL. It is clear that the accurate and comprehensive knowledge of the curved wall effects on the physics of supersonic/hypersonic SDTBL will lead to the development of flow control mechanisms on high speed vehicle design. This article focuses on the study of supersonic/hypersonic SDTBL under the influence of strong streamline concave curvature, which can lead to the determination of appropriate flow control tools and design optimization in high speed science.

\section{MESH GENERATION, FLOW SOLVER, AND BOUNDARY CONDITIONS}

Figure 2 (a) shows a schematic of the computational domain for the strong concave curvature, or Case 1 in Table 1 . The proposed geometry in Case 1 is based on the experimental study of Donovan et al. [5] and covers full spatial dimensions of the experimental model. The available experimental results, such as wall pressure and wall shear stress, are used for our RANS validation. Furthermore, the experimental boundary layer in [5] evolves along the streamwise direction; however, it shows a homogeneous spanwise condition,

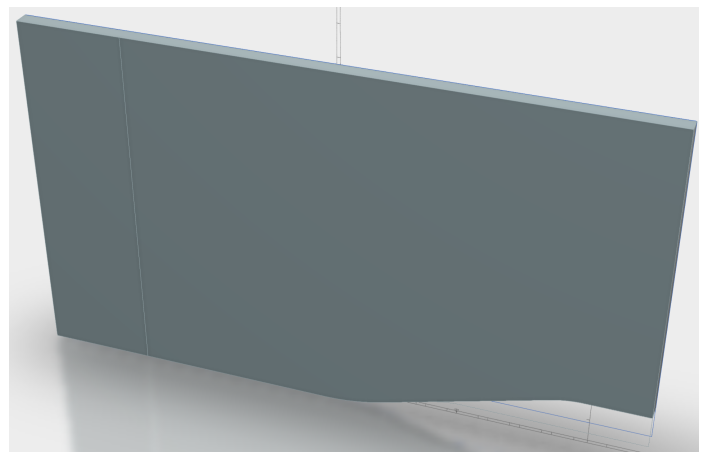

(a)

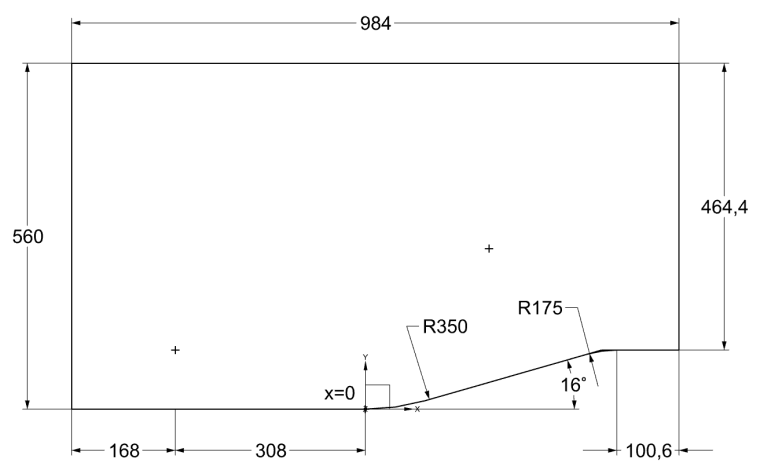

(b)

Figure 2: (a) Schematic of the computational domain, and (b) domain dimensions in $\mathbf{m m}$.

and consequently, the mean flow is two-dimensional. The wall curvature is prescribed based on the radius of curvature, $R$, defined in Table 1 in terms of the reference boundary layer thickness, $\delta_{r e f}$. Consistent with experiment, the value of $\delta_{\text {ref }}(=28 \mathrm{~mm})$ is taken at the origin of the coordinate system $(x=0 \mathrm{~mm}$ or beginning of the curved surface). Here, $x$ is the streamwise distance along the model surface, and $y$ is the wall-normal coordinate. The curved wall ends at $x=98 \mathrm{~mm}$, or approximately $3.5 \delta_{\text {ref }}$. The wall-curvature driven pressure gradient zone induces an Adverse Pressure Gradient (APG) on the flow. Upstream, there is a Zero-Pressure Gradient (ZPG) zone, which serves as a point of reference (i.e., baseline cases) to assess the effects of wall curvature on the flow. In Figure 2 (b), the corresponding $2 \mathrm{D}$ domain dimensions (in $\mathrm{mm}$ ) for Case 1 can be observed.

Table 1 summarizes the characteristics of the proposed two (2) cases according to the wall curvature $\left(\delta_{r e f} / R\right)$, Mach number $\left(M_{\infty}\right)$, momentum thickness-Reynolds numbers $\left(R e_{\theta}=\rho_{\infty} U_{\infty} \theta / \mu_{\infty}, R e_{\delta 2}=\right.$ $\rho_{\infty} U_{\infty} \theta / \mu_{w}$, based on the free-stream and wall viscosity, respectively), and computational domain dimensions in terms of $\delta_{r e f}$ (where subscript $\infty$ stands for free-stream values, $w$ stands for wall values, and $L_{x}$ and $L_{y}$ represent the streamwise and wall-normal domain lengths, respectively). Compressibility effects are taken into account by means of two different Mach numbers: 2.86 and 5, for cases 1 and 2, respectively, and by prescribing the same strong 
surface curvature, i.e. $\delta_{\text {ref }} / R=0.08$ in both cases. These two values for Mach numbers indicate that selected cases are in the supersonic regime and in the lower limit of hypersonic flows.

Flow Solver: The STAR-CCM+ package for computational fluid dynamics is selected to solve the governing equations of compressible flow in this investigation. The following turbulence models are prescribed for each case in Table 1: the Shear Stress Transport (SST) model by Menter [10] and the standard $k-\omega$ model by Wilcox [18]. Two-equation turbulence models are complete, because transport equations are solved for both turbulent scales, i.e. the velocity and the length scale. The original $k-\omega$ model [18] exhibits a freestream dependency of $\omega$, which is generally not present in the $k-\epsilon$ model. Menter [10] integrated the advantages of both models via blending functions, which permitted switching from $k-\omega$, close to a wall, to $k-\epsilon$, when approaching the edge of a boundary layer. A further improvement by Menter [10] was a modification to the eddy viscosity, based on the idea of the Johnson-King model, which established that the transport of the main turbulent shear stresses was crucial in the simulations of strong Adverse Pressure Gradient (APG) flows. This new approach was called the Menter shear-stress transport model (SST). In particular, the Menter SST turbulence model is wellknown for its good performance in boundary layer flows subjected to APG or flow deceleration. Since the concave surface curvature induces a strong deceleration on the flow, one of the purposes of the present study is to evaluate the SST's performance on concave walls.

Boundary Conditions: At the wall, the classical no-slip condition is imposed for velocities. Isothermal wall is assumed for the thermal field with $T_{w}=280.8 \mathrm{~K}$ as in [5]. The working fluid is calorically perfect non-reacting air. At the inlet boundary, four different options are tested and compared, which are described in detail in Section 3. At the top surface, freesstream values are prescribed.

Table 1: Numerical cases with concave surface curvature.

\begin{tabular}{|c|c|c|c|c|}
\hline Case & $\delta_{\text {ref }} / R$ & $M_{\infty}$ & $R e_{\theta} / R e_{\delta 2}$ & $L_{x} \times L_{y}$ \\
\hline 1 & 0.08 & 2.86 & $82,000 / 38,140$ & $35 \delta_{\text {ref }} \times 20 \delta_{\text {ref }}$ \\
\hline 2 & 0.08 & 5 & $143,360 / 66,679$ & $35 \delta_{\text {ref }} \times 20 \delta_{\text {ref }}$ \\
\hline
\end{tabular}

\section{NUMERICAL RESULTS}

For Case 1, the inlet free-stream velocity $U_{\infty}$ is set to $581 \mathrm{~m} / \mathrm{s}\left(M_{\infty}\right.$ $=2.86)$, whereas $U_{\infty}$ is set to $1014 \mathrm{~m} / \mathrm{s}\left(M_{\infty}=5\right)$ for Case 2 . In all cases, the static free-stream temperature $T_{\infty}$ is $102.4 \mathrm{~K}$. The grid is 723 (streamwise) by 200 (wall-normal) grid points. The mesh is stretched in the wall-normal direction with the first off-wall point located at $6 \times 10^{-8} \mathrm{~m}$. The first off-wall point in the Mach-5 case is placed at $\Delta y^{+} \approx 0.09$, which ensures an appropriate near wall resolution $\left(\Delta y^{+}<1\right)$. Figure 4 exhibits the time variation of flow residuals for continuity, momentum, energy, and turbulence transport (i.e., for turbulent kinetic energy, tke, and specific dissipation rate) equations. It is observed that numerical convergence is achieved in Case 1 by employing the SST turbulence model after 30,000 iterations with a CFL parameter of 0.5 .

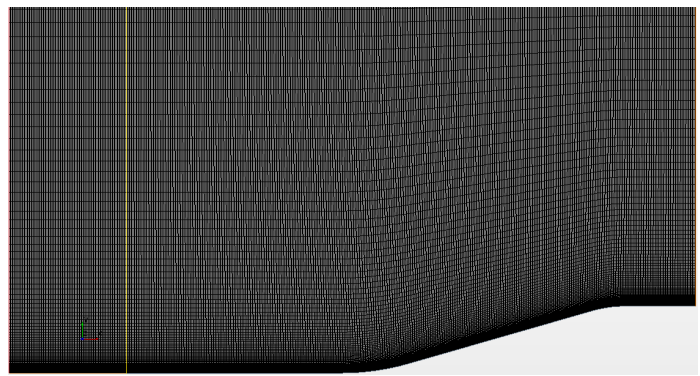

(a)

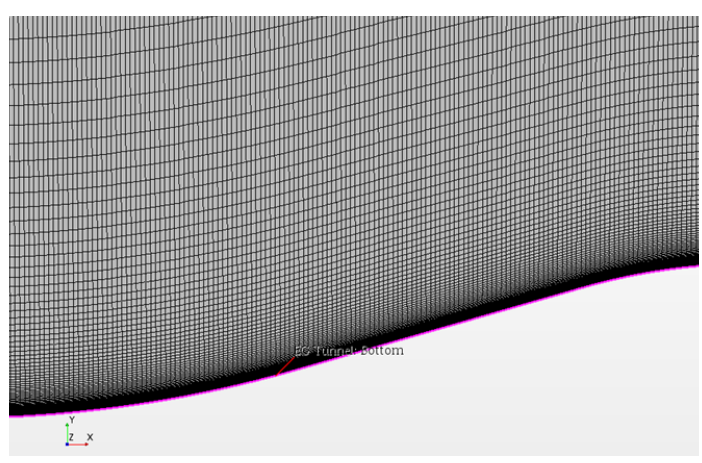

(b)

Figure 3: Computational mesh (a) total domain (flow from left to right) and (b) close-up of the curved surface.

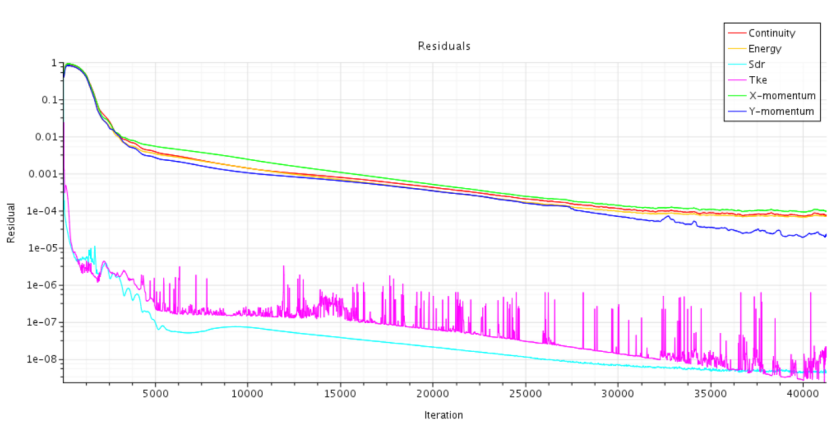

Figure 4: Time history of flow residuals.

Different methodologies for the inlet boundary condition are tested in Case 1. Figure 5 depicts iso-contours of the wall-normal velocity based on the SST turbulence model. In Figure 5 (a), the streamwise velocity $U$ was assigned a $1 / 7$ power law profile inside the boundary layer with a $U$-parabolic profile for the static temperature $T$. In Figure 5 (b) a composite velocity profile is prescribed that consists of Reichardt's [11] inner layer profile, Finley's wake function (Cebeci \& Bradshaw [4]), and the Walz equation for the temperature profile. In Figure 5 (c) the velocity and thermal profiles were extracted from $x=-0.0625 \mathrm{~m}$ in the previous case and reinjected at the domain inlet (this case is called "recycle"), whereas in 5 (d) a symmetry zone is attached upstream of the flat plate edge. 
Clearly, an inclined line of disturbances is observed in Figure 5 (a) at approximately $20^{\circ}$ with respect to the $x$-direction, very close to the Mach angle $\mu=\sin ^{-1}\left(1 / M_{\infty}\right)$, which is generated at the edge of the flat surface (no-slip condition) due to improper inlet flow profiles. This Mach wave is somehow reduced by setting the Reichardt's and Walz's equations at the inlet (see Figure 5 (b)), but not completely. The recycling technique in Figure 5 (c) has properly modeled inflow conditions, since the inclined disturbances almost disappeared. In a similar way, by attaching an upstream symmetry condition (see Figure $5(\mathrm{~d})$ ) and resolving turbulence transition, the sonic disturbances are minimized. However, in this case, the presence of the Mach wave is physical (not artificial) due to the interaction of supersonic free-stream with the edge of the flat plate. Therefore, the importance of setting realistic turbulent inflow conditions is unquestionable. Consequently, in the present study, an upstream slip boundary for modeling the inlet conditions is employed for the rest of the manuscript.

Figure 6 exhibits a comparison of present numerical results of Case 1 with the experimental wall static pressure. Generally speaking, both turbulence models (SST and $k-\omega$ ) capture quite well the significant increase (up to three times) of the upstream surface pressure due to the presence of the concave curvature (i.e. for $0 m \leq x \leq 0.098 m$ ). Beyond the end of the curved wall, more precisely in the inclined straight surface, some discrepancies with experimental values (in the order of 5\%) can be observed. However, the agreement is very good between numerical results for the SST model and inlet recycled profiles with Donovan's experimental data, particularly beyond the concave curvature (i.e. for $x>0.098 m$ ). This might be caused by a higher incoming Reynolds number prescribed in the inflow recycled profile case.

The skin friction coefficient $\left(C_{f, r e f}\right)$, defined as the wall shear stress normalized by the upstream free-stream density and velocity, is plotted in Figure 7. Experimental data from [5] is included. It can be seen that both turbulence models and inlet free-stream conditions exhibit similar performance. After the typical decreasing trend in the zero-pressure gradient region, numerical results of $C_{f, \text { ref }}$ based on the SST (free-stream inlet) and $k-\omega$ models significantly over-predict experimental values. On the other hand, the SST model (recycled profile inlet) shows an anomalous increasing trend of $C_{f, \text { ref }}$ in the ZPG zone; nevertheless, the obtained skin friction coefficient in the concave wall exhibits a much better agreement with experiments, which may be attributed to the higher Reynolds number imposed. Moreover, the observed increase in the skin friction coefficient is contrary to what occurs in incompressible boundary layers [2] subject to adverse pressure gradient, where the wall shear stress decreases. This is explained by the fact that the density increases more than the velocity decreases (flow deceleration) in compressible flows. Therefore, the boundary layer thickness based on the time-averaged streamwise velocity (not density-averaged) enlarges, as it will be shown later on, and the wall velocity gradient increases, as well. Figure 8 depicts the mean streamwise velocity at $x=40 \mathrm{~mm}$ (halfway through the curve) The SST and $k-\omega$ turbulence models (free-stream inlet) perform similarly with over-predictions of the order of $12 \%$ with respect to experiments from [5] at $y / \delta \approx 0.3$. On the other hand, the SST model (recycled profile inlet) exhibits a significant improvement when

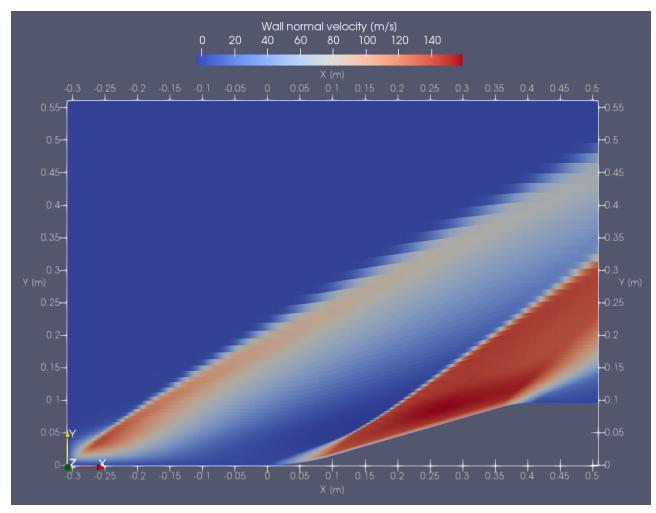

(a)

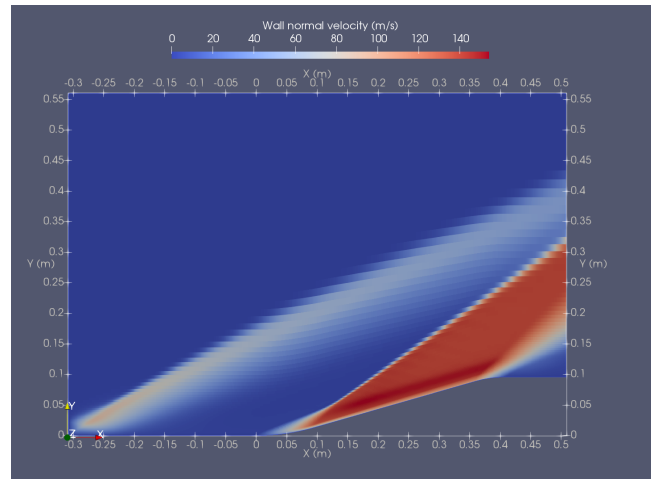

(b)

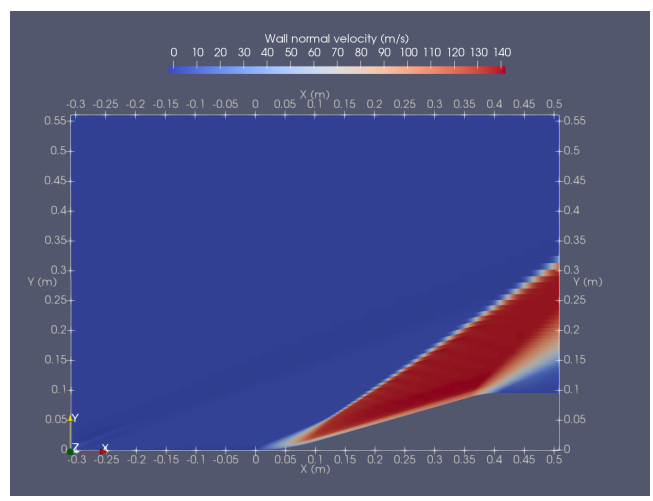

(c)

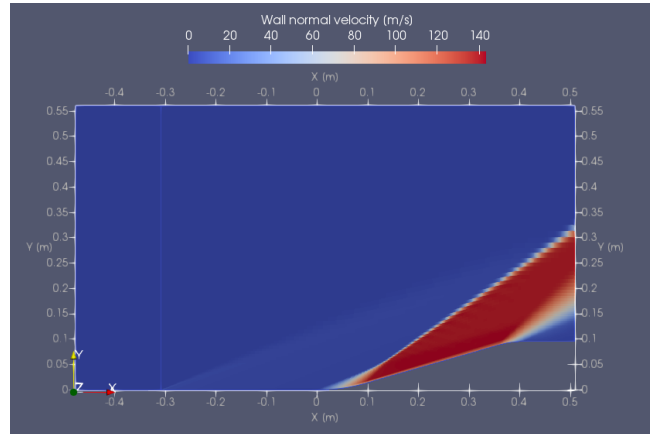

(d)

Figure 5: Iso-contours of wall-normal velocity for Case 1 and different inflow conditions. 


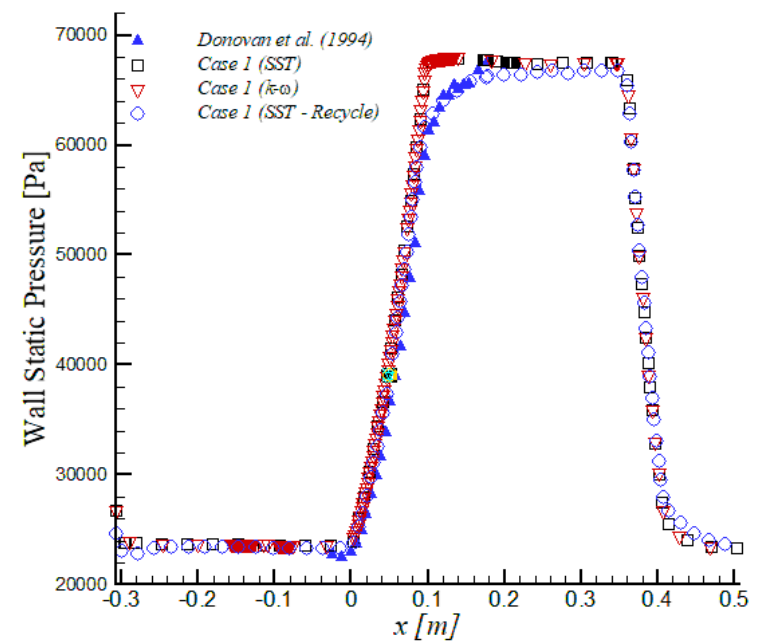

Figure 6: Wall static pressure.

the incoming Reynolds number is higher. A good agreement is observed with experimental data from [5], particularly for $y / \delta>0.25$. The mean temperature and mean streamwise velocity at $x=40$ $\mathrm{mm}$ (halfway through the curve) are plotted in Figure 9. In general, analogous trends of the analyzed three cases were observed when compared to the Walz's equation:

$$
\frac{T}{T_{\infty}}=\frac{T_{w}}{T_{\infty}}+\frac{T_{r}-T_{w}}{T_{\infty}}\left(\frac{U}{U_{\infty}}\right)-r \frac{\gamma-1}{2} M_{\infty}^{2}\left(\frac{U}{U_{\infty}}\right)^{2}
$$

where $T_{w}$ is the wall temperature, $T_{r}$ is the adiabatic or recovery temperature, $r$ is the recovery factor $\left(=\operatorname{Pr}^{1 / 3}\right.$, where $\operatorname{Pr}$ is the Prandtl number), and $\gamma$ is the specific heat ratio for air $(=1.4)$.

Figure 10 depicts the streamwise variation of the local boundary layer thickness for Case 1 by considering the SST model and inlet free-stream conditions vs. recycled profiles, respectively. The recycling method has generated much larger values of the inlet boundary layer thickness (i.e., $\delta \approx 20 \mathrm{~mm}$ ), very close to the experimental $\delta_{\text {ref }}$ of $28 \mathrm{~mm}$ as in [5]. On the contrary, the inlet free-stream condition has generated very small boundary layer thicknesses, indicating that a much longer ZPG zone would be necessary. It is inferred that, by means of this method, it is hard to control the desired inflow boundary layer thickness, requiring a significantly long inlet section to achieve the reference or target $\delta_{\text {ref }}$, and consequently, penalizing computational resources. Due to the presence of APG in the concave wall curvature from $x=0 \mathrm{~mm}$, the boundary layer significantly grows (up to $25 \%$ ). Downstream of the curved wall, the flow recovers and accelerates in the inclined ramp at a turning angle of $16^{\circ}$, where nearly constant values in the wall static pressure (see this ZPG region around $0.15 m<x<0.35 m$ in Figure 6) and in the boundary layer thickness are observed (see Figure 10). Beyond $x \approx$ $400 \mathrm{~mm}$, the convex wall curvature strongly accelerates the flow causing a relaminarization process. Additionally, a shrinking trend is seen for the boundary layer thickness along the convex wall. In Figure 11, a similar behavior of the $\operatorname{Re}_{\theta}$ streamwise variation can be seen. By the end of the concave curvature $(x \approx 98 \mathrm{~mm})$, the $R e_{\theta}$ has increased around 6 times regarding the incoming value.

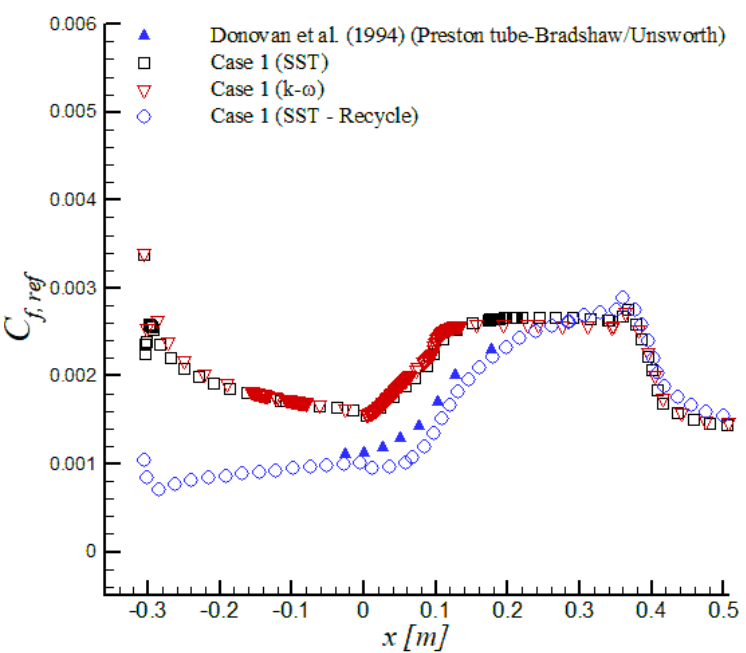

Figure 7: Skin friction coefficient.

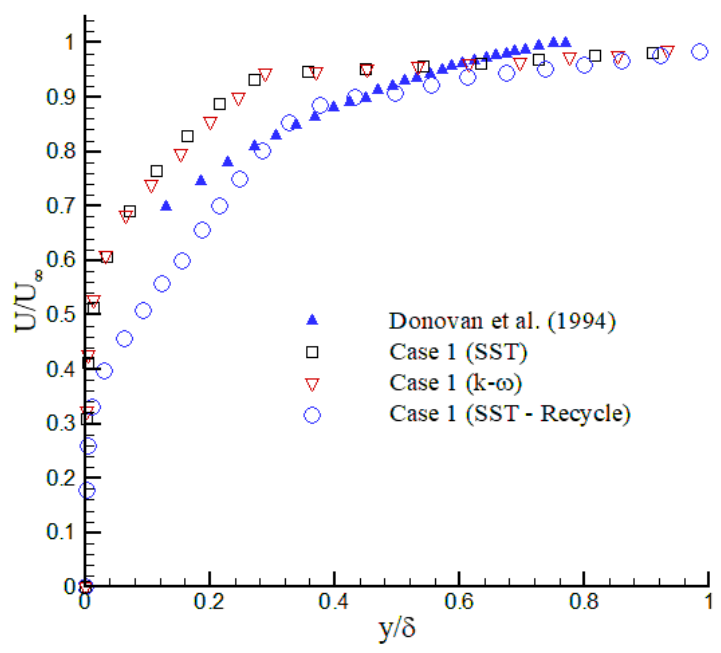

Figure 8: Mean streamwise velocity at $x=40 \mathrm{~mm}$ (halfway through the curve).

Figure 12 depicts iso-contours of streamwise velocity and Mach number for Case 1 and the SST turbulence model with inlet freestream conditions. At the inlet zone, the presence of a Mach cone inclined at approximately $20^{\circ}$ with respect to the streamwise direction due to flow perturbations (developing section) is nearly imperceptible. The lesson learned here is the importance of prescribing realistic turbulent inflow conditions. The strong concave wall curvature induces a significant deceleration on the flow or APG zone and formation of compression waves with decreasing values of the Mach numbers around 2. These compression waves formed a cone at approximately $35^{\circ}$, which are fully convected at the outlet plane without bouncing over the top surface. Therefore, future planned DNS studies on concave surfaces will have to consider at least $11 \delta_{r e f}$-tall computational domains. In spite of the 


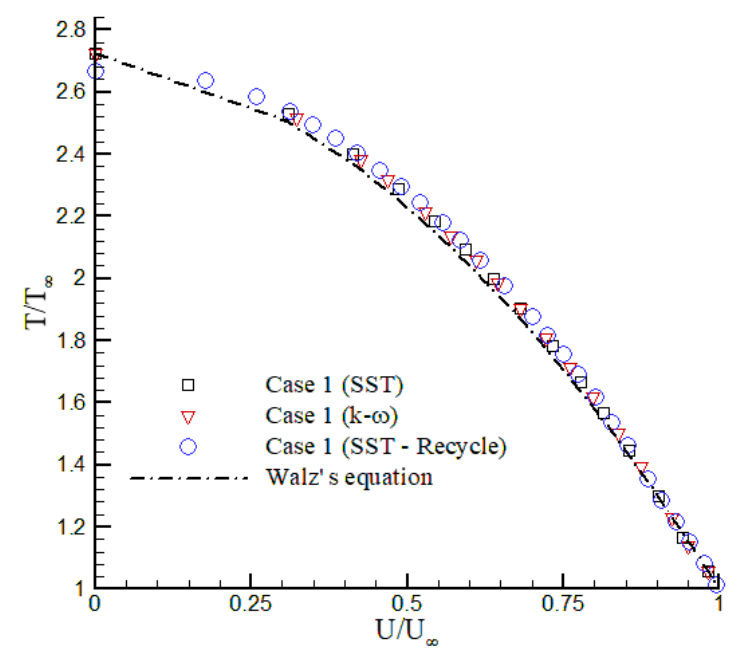

Figure 9: Mean temperature vs. mean streamwise velocity at $x=40 \mathrm{~mm}$ (halfway through the curve).

presence of a strong APG region, the flow remains fully attached in the curved-inclined wall region, confirmed by positive values of $C_{f, r e f}$ in Figure 7.

The static temperature is shown in Figure 13 (a). The flow experiences a thermal increase of roughly $70 \mathrm{~K}$ across the compression waves. Similar to the velocity boundary layer, it seems that the thermal boundary layer goes through a thickening process along the curved surface with an increase of the wall heat flux. From Figure 13 (b), it can be inferred that a strong concave curvature (with a curvature radius twelve times larger than the boundary layer thickness) may induce a compression ratio in the order of 2.5 at $M_{\infty} \sim 3$. In Figure 14, the effects of compressibility on the wall static pressure can be observed. In Case 2, the Mach number was set to 5 (hypersonic regime), and the selected turbulence model was SST with inlet free-stream conditions. The hypersonic regime provokes an approximate increase of $100 \%$ in the wall static pressure over the curved surface. Iso-contours of the Mach number in Figure 15 (a) show a more tilted (with respect to the streamwise direction $\left.\left(\sim 26^{\circ}\right)\right)$ and confined zone of compression waves at the hypersonic level. While the compression ratio is in the order of 6 for Case 2 (see Figure 15 (b)).

\section{CONCLUSIONS}

RANS simulations are performed in order to study the combined effects of wall concave curvature and compressibility. The computational domain is prescribed as in wind tunnel experiments by Donovan et al. [5]. In addition, a case is designed to shed some light on the influence of strong concave curvature and hypersonic speeds on the hydrodynamic/thermal field. The presence of strong curvature on spatially-developing turbulent boundary layers at Mach $=2.86$ induces an Adverse Pressure Gradient (APG) with a subsequent increase of the wall-shear stresses and wall-heat fluxes. The SST and $k-\omega$ turbulence models with free-stream inlet conditions have demonstrated similar performance when compared to experiments. A clear supremacy of the SST over the standard

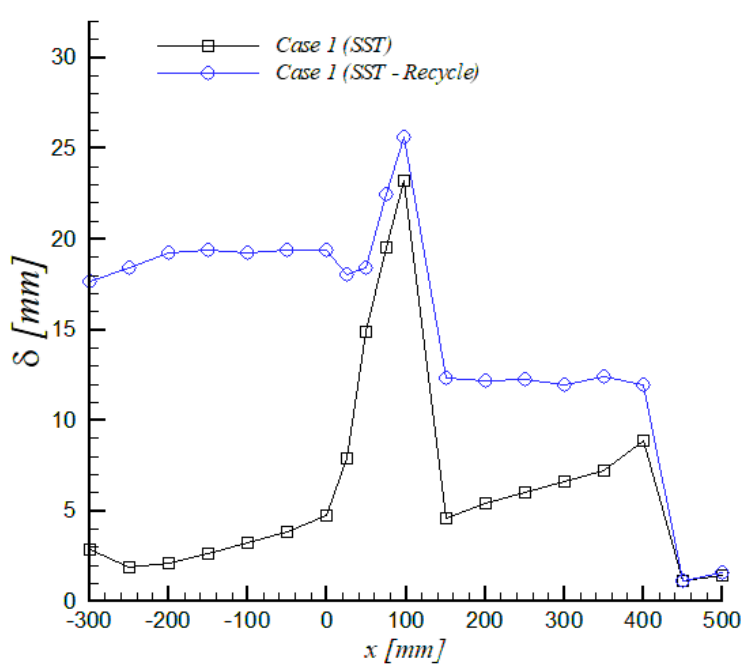

Figure 10: Streamwise variation of the boundary layer thickness $\delta$.

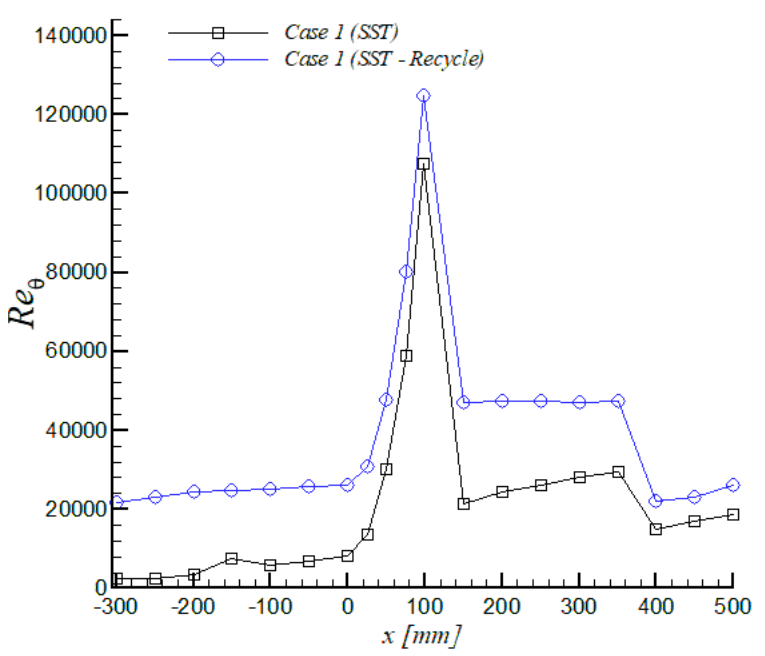

Figure 11: Streamwise variation of the Reynolds number $\operatorname{Re}_{\theta}$.

$k-\omega$ model has not been identified in the APG region, at least for the present conditions. However, the SST model based on recycled inflow profiles has shown a good agreement with experimental data by [5], since the incoming Reynolds number is higher. It has been estimated that a separate simulation of a flat plate turbulent boundary layer would add an extra $50 \%$ in terms of computational resources in order to extract, recycle, and inject the required inflow conditions to the principal domain. Nevertheless, and based on the quality of the obtained results, the extra resources pay off. The compression waves formed a $35^{\circ}$ cone; therefore, it is important to ensure enough room for the top surface in order to avoid wave reflections back to the domain. By increasing the inlet Mach number 


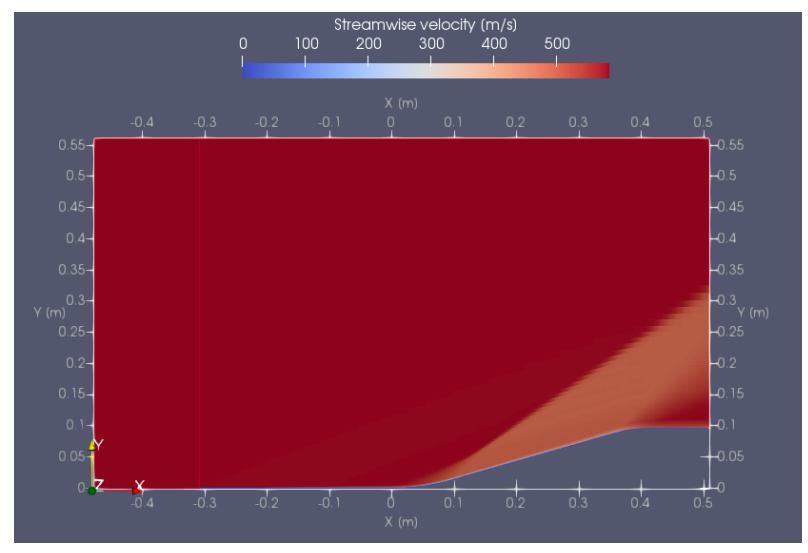

(a) Streamwise velocity

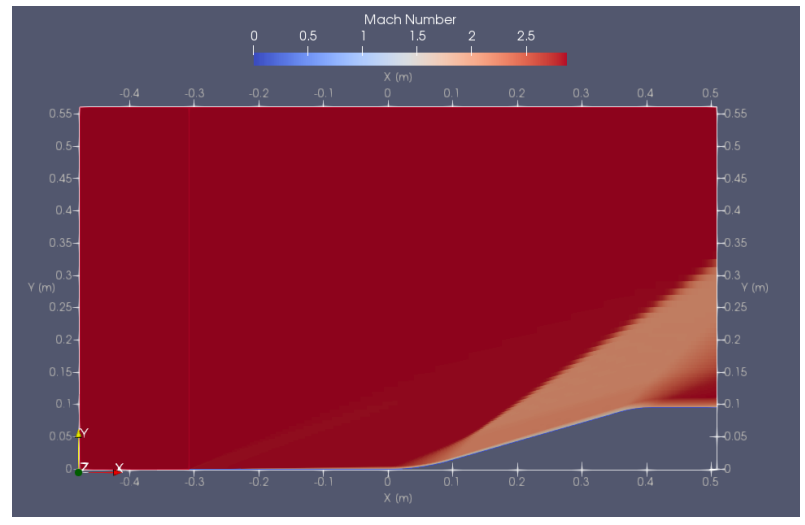

(b) Mach number

Figure 12: Iso-contours for Case 1 and SST turbulence model.

at the hypersonic regime, a more restrained compression zone has been observed with a significant increase in the compression ratio.

\section{REFLECTIONS}

I had little experience working with Computational Fluid Dynamics (CFD) prior to the Blue Waters Student Internship Program. For this reason, the experience has been a learning challenge and has broadened my knowledge in fluid dynamics, specifically in the area of supersonic and hypersonic flows. There were many challenges surrounding the underlying theory of fluid dynamics, specifically concerning the boundary layer in supersonic and hypersonic flows. Also, learning how to identify the cause of issues present in the simulation required persistence and in some cases creativity to resolve such issues. These issues usually highlighted areas where more learning was required. The internship experience helped me to learn more about turbulence and CFD.

\section{ACKNOWLEDGMENTS}

This material is based upon work supported by the Air Force Office of Scientific Research under award number FA9550-17-1-0051. ER acknowledges the Blue Waters Student Internship Program. This research is part of the Blue Waters sustained-petascale computing

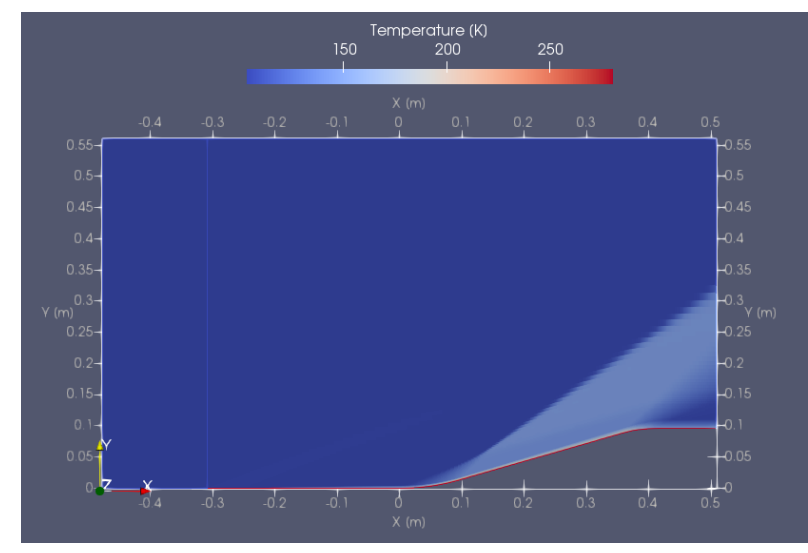

(a) Temperature

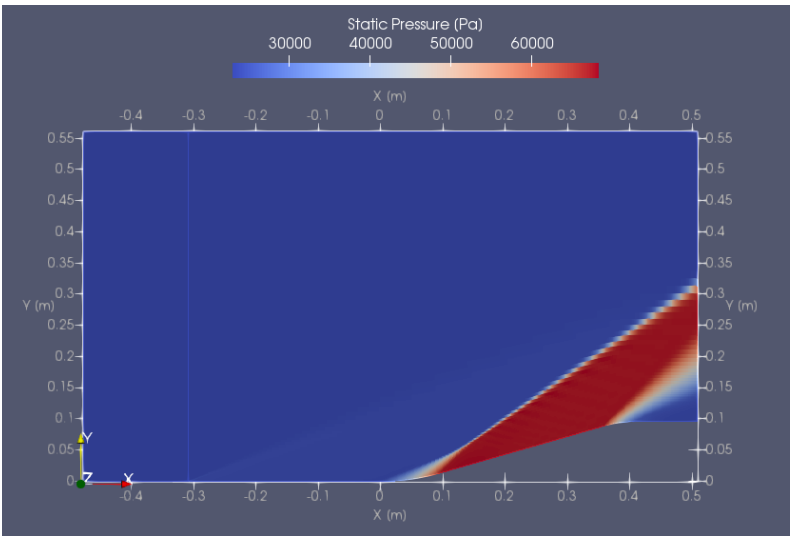

(b) Static pressure

Figure 13: Iso-contours for Case 1 and SST turbulence model.

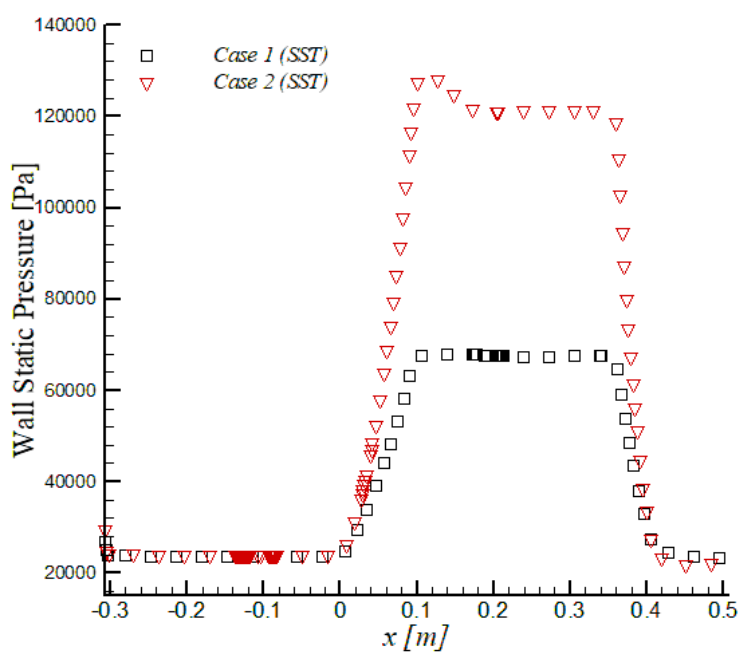

Figure 14: Wall static pressure. 


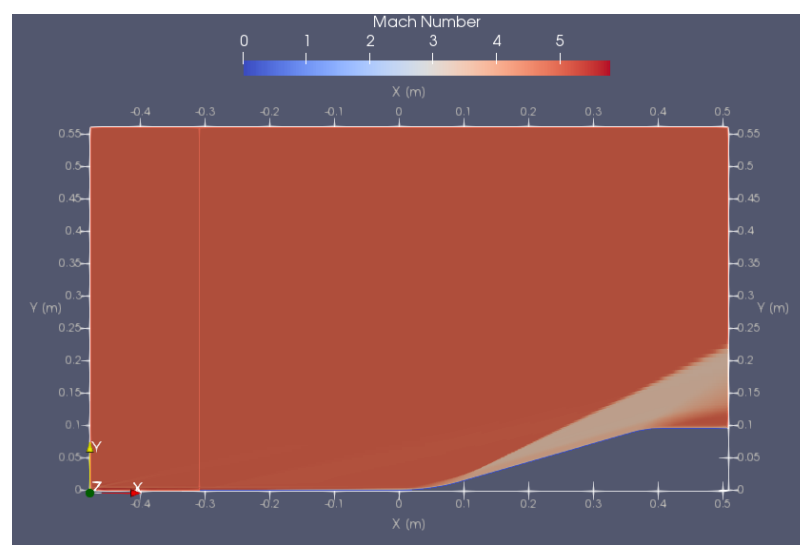

(a) Mach number

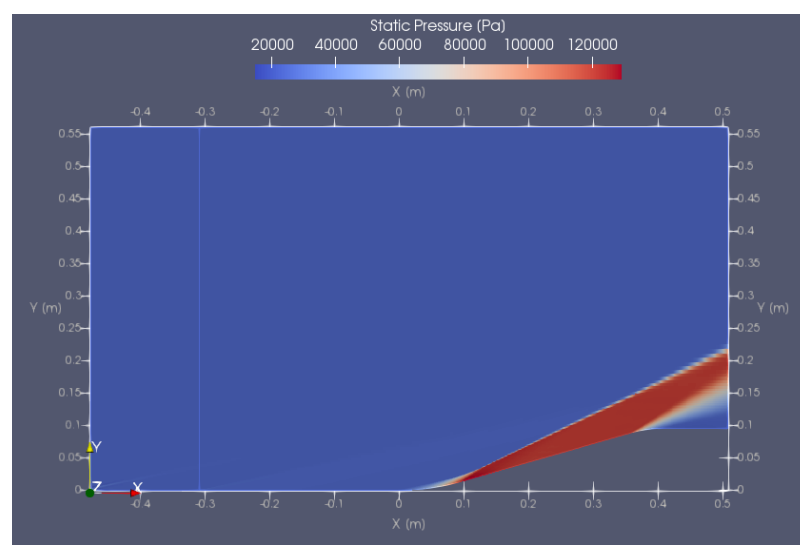

(b) Static pressure

Figure 15: Iso-contours of Case 2 (hypersonic) and SST turbulence model.

project, which is supported by the National Science Foundation (awards OCI-0725070 and ACI-1238993) and the state of Illinois. Blue Waters is a joint effort of the University of Illinois at UrbanaChampaign and its National Center for Supercomputing Applications.

\section{REFERENCES}

[1] Guillermo Araya and Luciano Castillo. 2013. DNS of Turbulent Boundary Layers Subjected to Adverse Pressure Gradients, In Progress in Turbulence and Wind Energy IV, Martin Oberlack, Joachim Peinke, Alessandro Talamelli, Luciano Castillo, and Michael Hölling (Eds.). Phys. Fluids 141, 187-190. https://doi.org/ 10.1007/978-3-642-28968-2 39

[2] Guillermo Araya, Luciano Castillo, Charles Meneveau, and Kenneth Jansen. 2011. A dynamic multi-scale approach for turbulent inflow boundary conditions in spatially developing flows. F. Fluid Mech. 670 (March 2011), 581-605. https: //doi.org/10.1017/S0022112010005616

[3] Peter Bradshaw. 1973. Effects of streamline curvature on turbulent flow. Technical Report AGARD-AG-169. Advisory Group for Aerospace Research and Development Paris (France).

[4] Tuncer Cebeci and Peter Bradshaw. 1977. Momentum Transfer in Boundary Layers. Hemisphere, Washington, DC.

[5] John F. Donovan, Eric F. Spina, and Alexander J. Smits. 1994. The structure of a supersonic turbulent boundary layer subjected to concave surface curvature. Journal of Fluid Mechanics 259 (January 1994), 1-24. https://doi.org/10.1017/ S0022112094000017

[6] Walter G. Hoydysh and Victor Zakkay. 1969. An experimental investigation of hypersonic turbulent boundary layers in adverse pressure gradient. AIAA fournal 7, 1 (January 1969), 105-116. https://doi.org/10.2514/3.5042

[7] Mohan Jayaram, Margaret W. Taylor, and Alexander J. Smits. 1987. The response of a compressible turbulent boundary layer to short regions of concave surface curvature. F. Fluid Mech. 175 (February 1987), 343-362. https://doi.org/10.1017/ S0022112087000429

[8] A. J. Laderman. 1980. Adverse Pressure Gradient Effects on Supersonic BoundaryLayer Turbulence. AIAA fournal 18, 10 (October 1980), 1186-1195. https: //doi.org/10.2514/3.50870

[9] George H McLafferty and Robert E Barber. 1962. The effect of adverse pressure gradients on the characteristics of turbulent boundary layers in supersonic streams. F. Aeronaut. Sci. 29, 1 (January 1962), 1-10. https://doi.org/10.2514/8.9294

[10] Florian R. Menter. 2009. Review of the shear-stress transport turbulence model experience from an industrial perspective. Int. F. of Computational Fluid Dynamics 23, 4 (May 2009), 305-316. https://doi.org/10.1080/10618560902773387

[11] H. Reichardt. 1951. Complete representation of the turbulent velocity distribution in smooth pipes. Z. Angew. Math. Mech. 31, 7 (January 1951), 208-219. https: //doi.org/10.1002/zamm.19510310704

[12] John D. Schmisseur. 2015. Hypersonics into the 21st century: A perspective on AFOSR-sponsored research in aerothermodynamics. Progress in Aerospace Sciences 72 (January 2015), 3 - 16. https://doi.org/10.1016/j.paerosci.2014.09.009

[13] Eric F. Spina, Alexander J. Smits, and Stephen K. Robinson. 1994. The Physics of Supersonic Turbulent Boundary Layers. Annual Review of Fluid Mechanics 26, 1 (January 1994), 287-319. https://doi.org/10.1146/annurev.fl.26.010194.001443

[14] Walter B. Sturek and James E. Danberg. 1972. Supersonic Turbulent Boundary Layer in Adverse Pressure Gradient. Part I: The Experiment. AIAA fournal 10, 4 (April 1972), 475-480. https://doi.org/10.2514/3.50122

[15] Walter B. Sturek and James E. Danberg. 1972. Supersonic Turbulent Boundary Layer in Adverse Pressure Gradient. Part II: Data Analysis. AIAA fournal 10, 5 (May 1972), 630-635. https://doi.org/10.2514/3.50167

[16] M. W. Taylor and A. J. Smits. 1984. The effect of a short region of concave curvature on a supersonic turbulent boundary layer. 22nd Aerospace Sciences Meeting (January 1984). https://doi.org/10.2514/6.1984-169

[17] H. Thomann. 1968. Effect of streamwise wall curvature on heat transfer in a turbulent boundary layer. F. Fluid Mech. 33, 2 (August 1968), 283-292. https: //doi.org/10.1017/S0022112068001308

[18] David C. Wilcox. 2006. Turbulence Modeling for CFD (3rd ed.). D C W Industries, La Canada, California. 